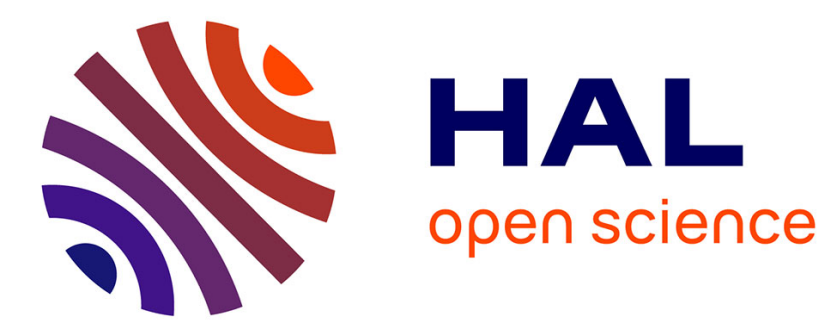

\title{
Michel BUTOR: un nouveau romancier sur les pas de Jean-Jacques Rousseau
}

Nicole Biagioli

\section{To cite this version:}

Nicole Biagioli. Michel BUTOR: un nouveau romancier sur les pas de Jean-Jacques Rousseau. Contemporary French and Francophone Studies, 2007, 11 (3), pp.369-378. hal-00151300

\section{HAL Id: hal-00151300 \\ https://hal.science/hal-00151300}

Submitted on 3 Jun 2007

HAL is a multi-disciplinary open access archive for the deposit and dissemination of scientific research documents, whether they are published or not. The documents may come from teaching and research institutions in France or abroad, or from public or private research centers.
L'archive ouverte pluridisciplinaire HAL, est destinée au dépôt et à la diffusion de documents scientifiques de niveau recherche, publiés ou non, émanant des établissements d'enseignement et de recherche français ou étrangers, des laboratoires publics ou privés. 
Contemporary French \& Francophone Studies, «Situating French » Roger Célestin, Eliane DalMolin, eds., David Ellison, guest co-editor, Vol 11, n³ 3-4, Fall/Winter 2007

\section{Nicole BIAGIOLI}

Centre Transdisciplinaire d'Épistémologie de la Littérature,

U.F.R. Lettres, Arts et Sciences humaines, 98 Bd. Édouard Herriot, B.P. 209, 06204 Nice CEDEX 3, France biagioli@unice.fr

et

Directeur de l'Équipe de Recherche en Technologie Educative n61, I3D (Interdidactique et discours des disciplines), Institut Universitaire de Formation des Maîtres Célestin Freinet-académie de Nice, 89 avenue

George V, 06046, Nice, CEDEX 1, France

\section{Michel BUTOR : un nouveau romancier sur les pas de Jean- Jacques Rousseau.}

\section{Résumé}

Errances botaniques: Lieux de mémoires (Peintures et dessins de Catherine Ernst), un des derniers ouvrages de M. Butor (2003), est à la fois un hommage indirect aux Rêveries du promeneur solitaire (1778) de Jean-Jacques ROUSSEAU et une refondation de la promenade littéraire. Les caractéristiques de ce genre, mixte d'autobiographie, de description botanique et de philosophie y sont exacerbées, interrogées et reconfigurées. Après une brève présentation historique, pragmatique et sémiotique du genre $\left(\mathrm{du} 18^{\text {ème }}\right.$ au $20^{\text {ème }}$ siècle), nous montrerons comment Butor se le réapproprie, en optant pour le tressage des sous-genres littéraires et iconiques qui le composent, plutôt que pour leur fusion.

Nous verrons ensuite comment il bouleverse la tradition littéraire, avec une écriture à deux voix et deux mediums, qui renverse la hiérarchie ordinaire du langage et de l'image illustrative. Le fait que les supports des gouaches florales soient des pages arrachées à deux ouvrages du $18^{\text {ème }}$, l'un juridique, l'autre religieux, renforce l'allusion à Rousseau tout en permettant de recadrer la tension entre les registres scientifique et poétique.

Se refusant à choisir entre les grands modes d'identification (animisme, naturalisme, totémisme et analogisme) de l'homme à son environnement, Butor rebranche le texte sur le monde, sans pour autant retomber dans le piège de la représentation, ce qui est une leçon d'anthropologie autant que de littérature.

Mots-clés : Butor, Jean-Jacques Rousseau, Nouveau Roman, autobiographie, promenade botanique

\section{Michel BUTOR : a «Nouveau Roman»'s author walking in Jean-Jacques Rousseau's footsteps.}

\section{Abstract}

Errances botaniques: Lieux de mémoires (Paintings and drawings by Catherine Ernst), one of M. Butor's last works (2003), is both a tribute indirectly payed to Jean-Jacques ROUSSEAU's Rêveries du promeneur solitaire (1778) and an attempt to renew the literary promenade. This genre, as a mixture of self-confidence, botanical description and philosophical essay, has its features overdrawn, examined and retrieved.

After a brief historical, pragmatical and semiotical account, we'll see how Butor planed to make his own Rousseau's inheritance. More particularly he would 'plait' rather than melt literary and pictural sub-genera which compose the botanical promenade as revisited by literature.

We'll see then how he overthrow traditions, using two voices and two mediums simultaneously and inverting illustrative common hierarchy between words and pictures. The fact that flowers are painted upon pages picked up from two $18^{\text {th }}$ century books, speaking one about law, the other about religion, enforce both the hint to Rousseau and the conflict between scientific and poetical tunes.

Not to choice between the great ways by which human beings identify themselves to what is surrounding (that is animism, naturalism, totemism, analogy) Butor successes in connecting again text and world, but without falling any more into the traps of representation. So doing, he gives us a magisterial lesson as well of literature and anthropology.

Keywords: Butor, Jean-Jacques Rousseau, Nouveau Roman, autobiography, botanical walk 
Contemporary French \& Francophone Studies, «Situating French » Roger Célestin, Eliane DalMolin, eds., David Ellison, guest co-editor, Vol 11, n³ 3-4, Fall/Winter 2007

Esquissé dès 1976 par Michel Launay ${ }^{1}$, le parallèle Butor-Rousseau a été renforcé a fil des ans par Butor lui-même: professeur à l'université de Genève, puis retiré près de la frontière suisse, proposant maintenant avec Errances botaniques Lieux de mémoires (2003), et Epîtres florales (2005) des récits d'herborisations.

Butor s'était déjà livré à des réécritures partielles de textes de Rousseau. Avec ces deux ouvrages il semble s'orienter désormais vers des reprises générique puisque leurs sources sont des œuvres qui ont contribué à créer ou à fixer un genre: les Rêveries du promeneur solitaire (1778), intertexte des Errances et archétype de la promenade littéraire; La Nouvelle Héloïse, (1761), intertexte des Epîtres qui marque un tournant dans l'histoire du roman épistolaire.

Errances botaniques est à la fois un hommage indirect aux Rêveries et une refondation de la rêverie botanique. Les caractéristiques de ce genre mixte y sont exacerbées, interrogées et reconfigurées. A travers lui, deux révolutions : 1789 (dont Rousseau est l'un des inspirateurs) et le Nouveau Roman (dont Butor est l'un des représentants) sont exhumées et critiquées. La passion botanique, trait d'union entre Rousseau et Butor, est un facteur de mise à distance et d'intégration dans la chaîne des êtres qui en fait un parfait intermédiaire entre la littérature et monde.

\section{L'héritage}

Entre les Errances et les Rêveries aucune ressemblance apparente. Le dernier ouvrage de Rousseau n'est pas illustré, Butor affiche l'image florale dès la couverture. Le titre de Rousseau ne fait pas allusion à la botanique, contrairement à celui de Butor. Seul le sous-titre de Butor lieux de mémoire confirme le désir de renouer avec la pratique commémorative de l'herbier instaurée par Rousseau:

Toutes mes courses de botanique, les diverses impressions du local des objets qui m’ont frappé, les idées qu'il m'a fait naître, les incidents qui s'y sont mêlés, tout cela m'a laissé des impressions qui se renouvellent par l'aspect des plantes herborisées dans ces mêmes lieux [...] je n'ai qu'à ouvrir mon herbier et bientôt il m'y transporte ( $7^{\text {ème }}$ promenade, 103).

Le rapport au lecteur change. Rousseau fait partager ses émotions, Butor ses cueillettes. Indéniable, la filiation est aussi complexe. 
Contemporary French \& Francophone Studies, «Situating French » Roger Célestin, Eliane DalMolin, eds., David Ellison, guest co-editor, Vol 11, n³ 3-4, Fall/Winter 2007

\section{1 La reprise}

En deux siècles la vieillesse a reculé de 60 à 80 ans mais elle est restée l'âge de la remémoration et de la mise en cohérence du schéma autobiographique. La botanique rappelle à Rousseau son «jeune âge» et «ses innocents plaisirs» (Septième promenade, 104), tandis que Butor se plaît à «herboriser comme le faisait le grand-père de [s]a grand-mère, et son père déjà peut-être en 1796, et qui sait ?son propre grand-père», Errances, 23).

Cet héritage culturel fait d'eux non des savants mais des amateurs. Loisir et passion («La botanique, telle que je l'ai toujours considérée, et telle qu'elle commençait à devenir passion pour moi, était précisément une étude oiseuse, propre à remplir tout le vide de mes loisirs», Confessions livre 12,55), l'herborisation leur offre la possibilité d' «errer»-le verbe est repris deux fois en une page dans la Septième promenade (98)- sans but mais non sans raison. En se promenant, l'herborisateur ne relie pas que des lieux, il articule aussi biotope végétal et paysage. Par le geste et le regard:

Je suis devant ce paysage éblouissant, ce glacier-ci, ces précipices, ces champs de neige [...] Je baisse les yeux, je regarde juste en face de moi à la hauteur de mes genoux et je me concentre sur une gentiane de Koch (Butor, ibid.)

Et par les outils qui les prolongent: pour Rousseau, «une pointe et une loupe sont tout l'appareil dont il a besoin pour observer» (ibid.). Depuis, l'équipement s'est sophistiqué mais Butor préfère aux «belles boîtes métalliques à herboriser» de son grand-père, un «carton qui a contenu des enveloppes pour [son] courrier» (ibid., 15). Leur pratique de l'herborisation est paratopique. Le concept de paratopie, «difficile négociation entre le lieu et le non-lieu, localisation parasitaire, qui vit de l'impossibilité même de se stabiliser» (Maingueneau, 1993, 28), inventé pour décrire le statut de la littérature dans la société, convient également à l'herborisation, à mi-chemin de la science et de la rêverie.

\section{2. La dénégation}

Dans les Errances l'attitude de Butor à l'égard de Rousseau est ambiguë. Il affiche les analogies mais laisse à son préfacier, Marcel Cottier, la responsabilité du rapprochement:

Comment ne pas songer, à travers cette façon originale d'herboriser, à celui qui a vu dans la nature non seulement un abri à l'écart de la société devenue pour lui menaçante, mais surtout un laboratoire permettant d'expérimenter de nouvelles sensations: comment ne pas songer à Jean-Jacques Rousseau[...] Il y a donc notre 
Contemporary French \& Francophone Studies, «Situating French » Roger Célestin, Eliane DalMolin, eds., David Ellison, guest co-editor, Vol 11, n³ 3-4, Fall/Winter 2007

célèbre citoyen de Genève, avec lequel Michel Butor établit une étroite parenté-nous invitant par là à une (re)découverte, au moins de la septième promenade[...] Mais il n’y a pas que lui. Bien d'autres textes résonnent sous les pas du poète [...] (9)

Sous une prudence de façade, celui-ci multiplie les sous-entendus. «A l'écart» est en effet le nom de la résidence de Butor, dont la plaque, qui fait allusion au prétendu élitisme du Nouveau Roman, est photographiée dans la biobibliographie des Epîtres florales.

Or dans les Epîtres florales le narrateur avoue: «je vais reprendre ma navigation dans La Nouvelle Hélö̈se, lecture obligée sur ces rives» (40). En deux ans, la crise de filiation a dû se dénouer...ou la stratégie narrative varier.

Les Errances sont donc placées sous le signe de la dénégation. Le titre démarque le mot-clef de la septième promenade «errer», mais le masque par le changement de catégorie lexicale. Du titre de Rousseau ne restent que la marque du pluriel et une allusion narquoise: «cela suffit à me faire skier sur les pentes de rêveries bien éloignées de la botanique» (p.18).

La relation intertextuelle est diluée par la substitution aux Rêveries de deux textes contemporains obscurs:

-Idée de la religion chrétienne, anonyme, Paris, 1723

-Essais sur l'état actuel de la France de B.A. Fonvielle 1796

dont les exemplaires abîmés, photographiés en annexe, sont décrits dans l'incipit:

J'emporte dans mon sac à dos, outre mon petit matériel de botaniste amateur, deux livres anciens trouvés dans un piètre état chez un brocanteur. Il manque de nombreuses pages et la reliure est écorchée.

Avant-dernière étape du déni puisque ceux-ci disparaissent à leur tour. L'illustratrice a en effet réalisé les paysages à l'encre sur des pages du traité religieux et les portraits de fleurs à la gouache sur celles de l'essai politique.

Le «repeint» est un changement apporté à un tableau après son achèvement, souvent par une main étrangère. Ici il porte sur l'écrit imprimé dont des fragments restent lisibles, prouvant que l'artiste a suspendu son geste par endroits. Il s'agirait donc plutôt d'un «repentir»: changement apporté à un tableau en cours d'exécution. De la lecture émerge une isotopie de la repentance («tout le mal vient de nous, et tout le bien vient de lui» (cahier 2), ainsi que le portrait, éclaté mais fidèle (8: «mais si j'ai abusé de cette liberté, si mon livre est nuisible, ne dois-je pas être puni»; 37: «âme véhémente, nourri de lieux communs de la philosophie il se 
Contemporary French \& Francophone Studies, «Situating French » Roger Célestin, Eliane DalMolin, eds., David Ellison, guest co-editor, Vol 11, n³ 3-4, Fall/Winter 2007

trouvait à sa place au milieu d'une révolution»; cahier 4: «car l'amour de la science rend les hommes superbes») d'un révolutionnaire repenti.

Après le rapprochement intertextuel confié au préfacier, c'est donc au tour de la métaphore métatextuelle, véritable marque de fabrique du Nouveau Roman, de se voir déléguée à l'illustratrice. La repentance évoque le geste qui a permis de la lire: le repentir pictural, et son auteur : C. Ernst. Mais ce geste de saccage et de résurrection, comme l'est tout geste révolutionnaire, renvoie aussi aux autres co-auteurs : Rousseau, inspirateur de 1789 et de Butor, et Butor, ex- nouveau romancier, mais aussi à en croire M. Launay, ex-soixantehuitard.

Cependant la fragmentation empêche la reprise du sens. Le«je» qui signifiait «on» redevient un pronom autobiographique en attente de sujet. L' «âme véhémente» ne désigne plus Mirabeau mais n'importe quel tribun. La phrase sur l'orgueil scientifique qu'on croirait empruntée à la carte $\mathrm{n}^{\circ} 11$ des brouillons des Rêveries: «je me représente l'étonnement de cette génération si superbe, si orgueilleuse, si fière de son prétendu savoir»- rappelle que Rousseau fut aussi un adversaire des Lumières. Il y a toujours plusieurs voix et plusieurs vérités chez tout individu et dans toute situation. Cette évidence est la seule qui soit révolutionnaire à condition d'être portée par le geste et non par le discours. Butor renouvelle la rêverie sur fond de révolution permanente.

\section{La N(ouvelle) R(êverie)}

Nouvelle Rêverie, Nouveau Roman, la coïncidence des initiales vaut pour les démarches. En acceptant de sortir du genre roman, Butor reste paradoxalement fidèle à l'esprit du Nouveau Roman, car s'il y a un clivage que le Nouveau Roman n'a pas subverti, c'est bien celui des genres. Il a exploré les limites du sien, mis en valeur la transversalité de certaines opérations d'écriture qui jusqu'alors semblaient plutôt réservées à la poésie, sans repenser le système fans sa globalité. Or c'est ce que fait Butor dans les Errances botaniques, qui ne sont pas à considérer comme une négation mais comme une extension du projet néoromanesque et peuvent donc être traitées avec les catégories imaginées par Ricardou pour analyser les procédures créatives du Nouveau Roman..

\section{1. Les renversements}

Comment un texte où l'illustration est omniprésente ${ }^{2}$, et qui obéit aux règles de représentation draconiennes de l'image scientifique (Biagioli, 217-231, 2006), peut-il être anti-représentatif? Trop de représentation tue la représentation. L' «enlisement descriptif» (Ricardou, 135, 1990) 
Contemporary French \& Francophone Studies, «Situating French » Roger Célestin, Eliane DalMolin, eds., David Ellison, guest co-editor, Vol 11, n³ 3-4, Fall/Winter 2007

consiste à détériorer le récit soit en hypertrophiant soit en désorganisant la description. La rêverie botanique entremêlant plusieurs codes, plusieurs registres discursifs et plusieurs mediums, l'enlisement descriptif y prend d'emblée une allure interdiscursive et intersémiotique.

En tant qu'objet naturel (Biagioli, 8, 1991) la fleur favorise la confrontation des codes et des discours. Les pères fondateurs de la botanique ont pris conscience de l'existence de l'effet de réel bien avant le Nouveau Roman (Biagioli, 131, 2000), lorsqu'ils ont compris que limitée aux seuls traits classificatoires (forme de la fleur, sexe, fruit), la description ne permettait plus de reconnaître les plantes et que, sans un peu de littérature et de dessin, la science ne pouvait fonctionner. Butor et son illustratrice retournent une première fois cette situation en remontant le coefficient «artistique» de l'illustration et de la description botaniques, puis une seconde fois en réinjectant du descriptif et de l'icône scientifiques entre et dans les passages picturaux ou littéraires.

Cette technique contrapuntique transforme l'enlisement descriptif en enlisement métadescriptif. Ce n'est plus la description qui est hypertrophiée mais son medium et son code. Le texte n'est pas paginé, mais les illustrations sont numérotées. Dans celles-ci, Cottier (préface, 7) note que «le réalisme de la technique picturale, fondé sur une observation et un rendu minutieux des plantes sélectionnées, est fortement contrasté- et de ce fait interrogé- par la liberté de traitement des fonds».

Quand il refuse d'illustrer l'expression «calice de la honte» par un cirse au motif «qu'il s'agit d'une composée, et [qu'] il faut parler de capitule pour la précision botanique», Butor feint de préserver la cohérence descriptive pour mieux compromettre la cohérence métadiscursive, oscillant entre science et littérature.

La rêverie botanique étant un genre descriptif, ce que n'est pas le roman, la description ne peut y être hypertrophiée que par rapport à elle-même. Butor va donc jouer sur l'intertexte et Les sous-genres descriptifs. Le descriptif scientifique, dominé chez Rousseau, devient dominant chez lui. Les planches botaniques sont complétées par un index plantarum et une bibliographie de la flore alpine. La rêverie contemplative, épisodique dans les Rêveries, est plus développée que la rêverie philosophique. Le narrateur l'assume alors qu'il se contente de citer les spéculations des contemporains de Rousseau.

Dans un genre fondé sur la digression («rêver»c'est étymologiquement «vagabonder») la mise en cause de l'unité descriptive ne peut être que contrastive. Butor rétablit la cohérence représentative là où Rousseau l'attaquait : dans le plan (quatre cahiers dans un ordre 
Contemporary French \& Francophone Studies, «Situating French » Roger Célestin, Eliane DalMolin, eds., David Ellison, guest co-editor, Vol 11, n³ 3-4, Fall/Winter 2007

chronologique contre dix promenades a-chronologiques), et l'affaiblit où il la renforçait, c'està-dire dans le texte. Plus de suture autobiographique entre passages réflexifs et passages descriptifs: le narrateur butorien s'énonce mais ne se raconte pas. Plus de leit-motiv: sa solitude est conjoncturelle, pas fatale. En revanche, il multiplie et commente les citations des deux contemporains de Rousseau là où ce dernier ne faisait que mentionner ses propres oeuvres (Confessions, Dialogues). Rousseau s'efforçait de rassembler ses voix intérieures, Butor s'ouvre à la polyphonie du discours social, en commençant malicieusement par celui que son modèle refusait d'écouter.

\section{2. L'errance comme conséquence}

Après le renversement de l'ordre institué, le Nouveau Roman assignait au récit la tâche paradoxale de se raconter tout en racontant. La création se déroulant dans le temps, seul un récit peut en rendre compte. Il faut donc, si l'on étend la révolution textuelle à un genre descriptif, y introduire du récit et du récit autotélique. «La mécanisation du récit, avec l'effet d'exhibition qui en découle, nul nouveau romancier ne l'a sans doute poussée plus loin que Michel Butor» reconnaissait Ricardou en 1973 (50). Presque un demi-siècle après L'emploi du temps et La modification, Butor met les dispositifs néo romanesques de ses débuts au service de ce qu'il est devenu : un auteur polyvalent.

La forme la plus directe d'autoreprésentation est la mise en abyme. Butor entrecoupe son récit d'herborisation de poèmes, dizains pentamétriques en vers libres, qui donnent la parole aux fleurs. Des fleurs se décrivant au milieu d'un récit qui décrit la découverte d'autres fleurs, la redondance sémantique est forte, mais ne doit pas masquer la différence énonciative. Dans la promenade, la fleur est objet de discours; dans la prosopopée poétique, elle est sujet de l'énoncé et de l'énonciation:

Odorante et vive

entre les pierrailles

j'étale mes lèvres

en dressant mon casque

pour te murmurer

botaniste en herbe

les secrets du vent

sortant des fissures

après ton passage au creux du torrent (Sarriette des Alpes, 16) 
Contemporary French \& Francophone Studies, «Situating French » Roger Célestin, Eliane DalMolin, eds., David Ellison, guest co-editor, Vol 11, n³ 3-4, Fall/Winter 2007

Bien que descriptifs, les poèmes sont des métaphores du récit car ils retracent une aventure: celle de l'échange des voix du sujet et de l'objet dans l'énonciation lyrique. Simple interlocuteur des poèmes, le poète n'est pas identifié au narrateur dans les passages en prose ${ }^{3}$. Il est donc sans voix. Quant au narrateur, il perd la sienne et la retrouve chaque fois qu'il cite, sauf à la fin:

Si mon livre échappe à l'oubli, s'il survit à nos troubles comme document historique...»Il n'a pas échappé à l'oubli. Quant au mien...Nous approchons du village.

où il reprend l'interrogation d'un auteur du passé pour interpeller le lectorat à venir. Les seize poèmes sont des mises en abyme à la fois rétrospectives de la «disparition élocutoire» du poète, et prospectives de celle du narrateur. Ils sont ainsi désignés comme pivot illocutoire de l'œuvre, ce qui invite à considérer plus attentivement leur place. On découvre alors une symétrie compositionnelle réglée par l'alternance prose /poésie:

\begin{tabular}{|llll|llllll|}
\hline $\mathrm{C} 1$ & 3 & 4 & 2 & $\mathrm{C} 2$ & 2 & 2 & 1 & 2 & 2 \\
& $\mathrm{R}$ & $\mathrm{P}$ & $\mathrm{R}$ & & $\mathrm{R}$ & $\mathrm{P}$ & $\mathrm{R}$ & $\mathrm{P}$ & $\mathrm{R}$ \\
\hline $\mathrm{C} 3$ & 3 & 4 & 2 & $\mathrm{C} 4$ & 2 & 2 & 1 & 2 & 2 \\
& $\mathrm{R}$ & $\mathrm{P}$ & $\mathrm{R}$ & & $\mathrm{R}$ & $\mathrm{P}$ & $\mathrm{R}$ & $\mathrm{P}$ & $\mathrm{R}$ \\
\hline
\end{tabular}

$(\mathrm{C}=$ Cahier, $\mathrm{R}=$ récit, $\mathrm{P}=$ poème $)$

A générateur puissant, récit dégénéré. Du sommaire reconstitué à partir des titres des cahiers émerge un scénario-type:

Cahier 1 Le sentier (Val d'Hérens, Alpes valaisannes)

Cahier 2 La pause (Val d'Hérens, Alpes valaisannes)

Cahier 3 La récolte (La Gruyère, Alpes fribourgeoises)

Cahier 4 Le retour (Le Lötschental, Alpes valaisannes)

récusé à mi-parcours dans l'explicit du deuxième cahier:

Mais je suis là, je suis bien là, dans le val d'Hérens, au cœur des Alpes valaisannes. La semaine dernière j'étais dans la Gruyère, c'étaient les Alpes fribourgeoises. La semaine prochaine, j'ai projet de parcourir le Lötschental, une autre partie du Valais. 
Contemporary French \& Francophone Studies, «Situating French » Roger Célestin, Eliane DalMolin, eds., David Ellison, guest co-editor, Vol 11, n³ 3-4, Fall/Winter 2007

L'herborisation en Gruyère (3) se révèle antérieure à celles du Valais (1 et 2). Le périple n'est donc qu'une fiction de présentation. L'ordre des cahiers n'est pas chronologique mais symbolique. Aboutissement logique du dispositif anti-représentatif, la vérité bascule du côté de l'isotopie métatextuelle. «Je m'adapte à la pente, à la texture» est le seul énoncé véridique de tout l'ouvrage (index scientifique exclu). Il est vrai aussi bien métaphoriquement que littéralement, du texte (qui s'autodésigne à travers la fiction), et de l'image (la pente de la montagne derrière le chardon de l'illustration voisine laissant voir le fonds imprimé). Placé au début (devant l'illustration 10 sur 37), il désigne localement et globalement le fonctionnement amphibologique d'un dispositif qui se refuse à choisir entre fiction et réalité, texte et image, présent et passé.

\section{Une anthropologie littéraire}

Quelle sorte de repenti est donc Butor s'il ne renie ni l'histoire ni les techniques du Nouveau Roman?

En fait il ne se désolidarise que de ses excès et des effets pervers de sa contre-censure. Le Nouveau Roman, en brisant des tabous, en a créé d'autres, autour de la représentation et de l'expression (Biagioli, 318, 2005), discréditant non seulement le roman traditionnel mais aussi les genres liés à la subjectivité, particulièrement l'autobiographie et la poésie.

La position de Butor est celle d'un casuiste, qui travaille à concilier subjectivité et textualité, en s'appuyant sur deux intermédiaires: l'intertextualité et la devise formée par l'association du poème et de l'image florale. Dans la citation comme dans la devise, la dissociation du lieu de l'énonciation, de la source énonciative et de l'énoncé a pour effet de bloquer la réflexivité discursive. L'une emprunte la parole, l'autre la prête, ce sont les deux pôles du courant communicationnel. Mais le sens s'y déplace sans prendre consistance parce que le sujet ne s'y engage pas.

A l'inverse, la science et la littérature font partie des discours assumés. La responsabilité de l'émetteur y favorise la cohérence interne de l'énoncé, lequel en retour se détache de son énonciateur. L'autonomie du texte est un trait de ressemblance entre elles qui tend à s'accentuer chaque fois que l'une ou l'autre réfléchit aux conditions de sa communication, cas de la botanique classique comme du Nouveau Roman. C'est pourquoi aussi littérature et science peuvent collaborer.

En bon héritier des Rêveries, Butor exploite les propriétés euphorisantes de l'herborisation découvertes par Rousseau, tout en évacuant les scories de l'autobiographique: regrets, 
Contemporary French \& Francophone Studies, «Situating French » Roger Célestin, Eliane DalMolin, eds., David Ellison, guest co-editor, Vol 11, n³ 3-4, Fall/Winter 2007

plaintes, autojustification. Mais son originalité réside surtout dans sa volonté d'explorer, après le fonctionnement interne des genres, leur fonctionnement externe, c'est-à-dire les mécanismes qui règlent leur coexistence.

Comme pratique culturelle, la littérature est parcourue par les quatre modes d'identification possibles de l'homme avec son environnement (Descola, 66):

\begin{tabular}{|l|l|l|}
\hline physique & Continuité & Différence \\
\hline Continuité & totémisme & science \\
\hline Différence & animisme & analogie \\
\hline
\end{tabular}

Aucune de ces tendances ne peut être durablement censurée sans danger pour l'équilibre global de la conscience et de la culture. Dans les Errances botaniques, animisme (la fleur parle) ; science (on la classe) ; totémisme (elle donne accès au clan des herborisateurs); analogie (on la métaphorise) ne sont pas confondus, ni opposés, mais confrontés. Butor fonde son anthropologie littéraire sur une extension polyphonique de l'antireprésentation.

\section{Notes}

(1) p. 553 : «Butor est le Rousseau des temps modernes [...]. Quoi, s'écriera-t-on, oser comparer un écrivain peu engagé, un formaliste, un jongleur, au prophète qui a dénoncé l'imposture du monde corrompu[...]? Oui, Butor et peut-être l'écrivain du vingtième siècle qui dénonce avec le plus de force la corruption, la pourriture de ce monde malade».

(2) 48 gouaches, 18 dessins au crayon pour les portraits de fleurs, 1 photo noir et blanc grand format pour une nature-morte florale (bouquets dans des vases), 4 encres couleurs, 4 encres noires, 3 photos noir et blanc grand format et 20 photos noir et blanc petit format pour les paysages.

(3) Il sera identifié rétrospectivement par une confidence du narrateur des Epîtres florales: «je me hasarde aussi à tracer des poèmes dans lesquels je m'abstiens de rimer» (p.124), quoique rien ne soit dit sur le contenu des poèmes, qu'on suppose floral en raison du contexte. 
Contemporary French \& Francophone Studies, «Situating French » Roger Célestin, Eliane DalMolin, eds., David Ellison, guest co-editor, Vol 11, n³ 3-4, Fall/Winter 2007

\section{BIBLIOGRAPHIE}

BIAGIOLI Nicole, Sémiotiques de la fleur, thèse de doctorat d'état, Lyon 2, 1991.

BIAGIOLI Nicole, «Censure et Nouveau Roman» dans Censure, autocensure et art d'écrire, éd. Jacques Domenech, Complexes, 2005.

BIAGIOLI Nicole, «Le trait botanique dans l'œuvre de Michel Butor» dans Le trait, langue/visage/paysage, de la lettre à la figure, éd. B. Bonhomme, L'Harmattan, 217-231, 2006.

BIAGIOLI Nicole, «Description scientifique et didactique: le cas de la botanique», Enjeux revue de didactique du français «La description», $n^{\circ}$ 47, Namur, 2000.

BUTOR Michel, ERNST Catherine, ERRANCES BOTANIQUES, Slatkine, Genève, 2003.

BUTOR Michel, ERNST Catherine, EPITRES FLORALES, Slatkine, Genève, 2005.

DESCOLA Philippe, «Le monde par-delà la nature et la culture», La Recherche 374, avril 2004.

LAUNAY Michel, «Rousseau, Butor et les ordinateurs», dans Gilbert Fauconnier et Michel Launay, Index-concordance des Rêveries du promeneur solitaire, Slatkine-Champion, 1978 MAINGUENEAU Dominique, Le contexte de l'oeuvre littéraire, Dunod, 1993.

RICARDOU Jean, Le Nouveau Roman, Seuil, 1973,1990.

ROUSSEAU Jean-Jacques, Confessions t. 2, Livre de poche, éd.1972.

ROUSSEAU Jean-Jacques, Les Rêveries du promeneur solitaire, Garnier, Paris, éd.1960. 